\title{
Physico-chemical treatment methods for the removal of microcystins (cyanobacterial hepatotoxins) from potable waters
}

\author{
Linda A. Lawton and Peter K. J. Robertson \\ School of Applied Sciences, The Robert Gordon University, St Andrew Street, Aberdeen, UK \\ $A B 251 H G$
}

Received 6th January 1999

The incidence of cyanobacterial blooms in freshwaters, including drinking water reservoirs, has increased over the past few decades due to rising nutrient levels. Microcystins are hepatotoxins released from cyanobacteria and have been responsible for the death of humans as well as domestic and wild animals. Microcystins are chemically very stable and many processes have only limited efficacy in removing them. In this paper we review a range of water treatment methods which have been applied to removing microcystins from potable waters.

\section{Introduction}

Cyanobacteria (blue-green algae) produce several types of toxins that can be harmful to humans. The most frequently occurring are the microcystins, a group of at least 60 heptapeptides which share the common structure, cyclo(D-AlaL-X-erythro- $\beta$-D-methylaspartic acid-L-Y-ADDA-D-isoglutamic acid- $N$-methyldehydroalanine), where $\mathrm{X}$ and $\mathrm{Y}$ are variable $\mathrm{L}$ amino acids and ADDA is a unique 20-carbon amino acid (3-amino-9-methoxy-2,6,8-trimethyl-10-phenyldeca-4,6-dienoic acid). Microcystins are named according to the variable amino acids that they contain. Microcystin-LR (Fig. 1), one of the most commonly occurring and frequently studied microcystins, contains leucine (L) and arginine (R) in the variable positions. It has been established that microcystin toxicity is due to their potent and irreversible inhibition of important regulatory enzymes (protein phosphatase 1 and 2A) which can cause both acute and chronic effects. Of great concern is the tumour promoting activity of microcystins and hence there have been recent moves to minimise exposure through the publication of a guideline value $(1 \mu \mathrm{g} \mathrm{dm}-3$ in potable water) by the World Health Organisation. A full discussion and extensive
Dr Linda Lawton is a Lecturer in Environmental Science within the School of Applied Sciences of The Robert Gordon University. She has eleven years experience in the field of toxic cyanobacteria with her key areas of expertise in the chemical analysis of cyanotoxins including extraction, purification and chemical characterisation. Over a number of years she has focused much of her attention on the detection and removal of cyanotoxins from potable water including the development of non-mammalian bioassays to determine the toxicity of degradation products. Currently much of her research effort, in conjunction with Dr Peter Robertson, has been targeted towards the use of photocatalysis as potential treatment method for the removal of microcystins from drinking water. Dr Lawton is Secretary to the Department of the Environment's Standing Committee of Analysts, Toxins Panel (6.11) and has recently

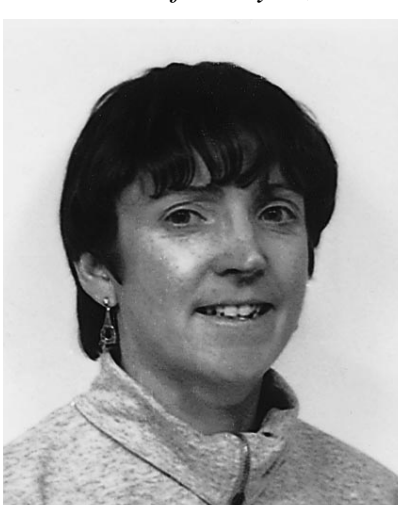

Linda A. Lawton
On completing his D.Phil. at the University of Ulster in October 1989 Dr Peter Robertson joined the Faraday Centre in Carlow, Ireland. In this post he was involved in a wide range of electrochemical research projects including bulk electrolysis processes and environmental treatment systems. In $1991 \mathrm{Dr}$ Robertson moved to the Industrial Research and Technology Unit just outside Belfast where he was a Higher Scientific Officer. This government body acted as an industrial consultancy and research unit for government and industry in Northern Ireland. In this position he managed a range of research projects on photocatalytic and electrochemical waste treatment for local industry. Dr Robertson also assisted several companies in solving contamination problems that they were experiencing and aided them with product developments. Dr Robertson joined the staff of the School of Applied Sciences

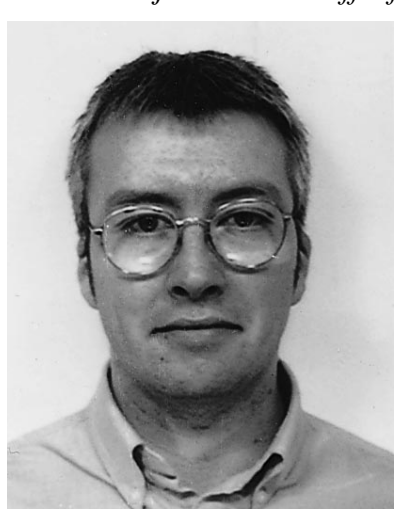
at Robert Gordon University in September 1995. The main areas of his research centre around photocatalytic treatment of potable water and industrial waste effluents, heavy metal recovery and homogenous photocatalysis. He is also involved in the development of heavy metal and hydrocarbon sensors for the marine, estuarine and fresh water environments. 


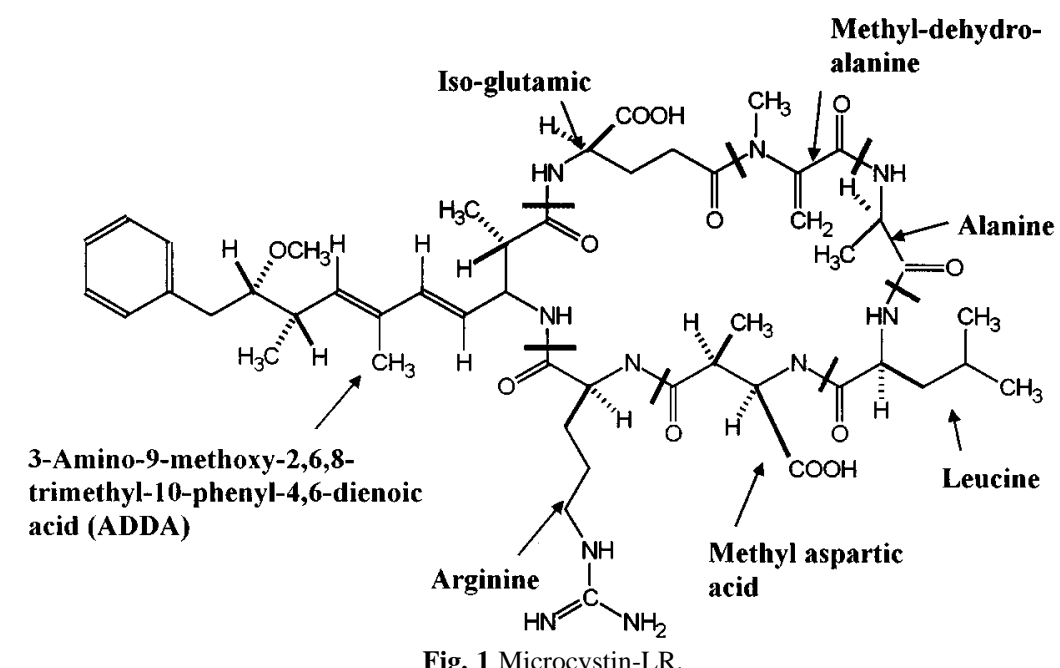

Fig. 1 Microcystin-LR.

referencing of many aspects of microcystins can be found in reference 1.1

Cyanobacteria typically occur in high numbers (often referred to as a bloom) in nutrient-rich water bodies. These blooms are usually found in lakes and reservoirs, but more recently very slow flowing rivers have also been affected. Increasing awareness and/or occurrence has focused attention on the potential impact on human health of microcystins in drinking water. Furthermore, the recent fatalities of dialysis patients ${ }^{2}$ due to contaminated water highlight the essential need for reliable treatment and monitoring methods.

Microcystins are known to be relatively stable compounds, possibly as a result of their cyclic structure. They are reported to withstand many hours of boiling and may persist for many years when stored dry at room temperature. It is therefore not surprising that microcystins are not readily removed from drinking water by conventional treatment methods. The investigation of suitable methods for their removal spans over twenty years, beginning even before microcystins were structurally characterised. It is only in recent years that the use of a broad range of advanced techniques for toxin removal has been applied and evaluated.

Microcystins are produced and retained within healthy cyanobacterial cells and are only released into the surrounding water when cells die and break open. They can, therefore, enter water treatment works either as dissolved compounds in the raw water or within cells. Partitioning of this nature presents a unique challenge to water treatment processes. Removal of toxin containing cells at the early stages of treatment can be a relatively straightforward way of greatly reducing the microcystin burden. In doing so however, great care must be taken to prevent cell lysis or toxins will move into the water phase and the benefit is negated. In contrast, it may be more desirable to release microcystins into the liquid phase where they can be removed by treatment processes. Where whole cells are permitted to pass through the treatment system, the toxins retained within may be protected from adsorption or chemical destruction. These issues are primarily a system management problem and water treatment engineers must be aware of how cells behave within individual treatment plants. For the purpose of this review we will focus on the removal of microcystins dissolved in water.

\section{Physical treatment methods for the removal of microcystins}

Once the stability and resistance to traditional treatment methods had been established, the use of alternative methods for the removal of microcystins was investigated. Early treatment studies focused primarily on the physical removal of microcystins by adsorption technologies, particularly the application of activated carbon, with detailed studies into the use of this technique continuing to the present day. Other removal methods including reverse osmosis have also been assessed.

\subsection{Activated carbon}

Two general types of activated carbon are utilised by the water industry for the removal of trace organics. Granular activated carbon (GAC) is typically used in flow-through column reactors whereas powdered activated carbon (PAC) can be added directly to water prior to coagulation or filtration. The removal of taste and odour problems, also caused by cyanobacteria, were one of the initial applications of activated carbons in the water industry with their use now expanded to a wide range of contaminants.

Systematic studies by Hoffman in 1976 revealed that flocculation/sedimentation, sand filtration and chlorination, all failed to eliminate the toxicity associated with the peptide toxins from cyanobacteria. ${ }^{3}$ The same study, however, repeatedly found that filtration through activated carbon was capable of removing the toxins, as was the addition of PAC, although this was dependent on dosing levels. The required dosing rate appeared to vary depending on the microcystin variant under investigation, but no further elucidation regarding the factors influencing this mechanism can be made since the toxins were uncharacterised at the time. Removal of both microcystins under investigation was achieved at $800 \mathrm{~g} \mathrm{~m}^{-3}$ which was a relatively high PAC dosing rate (typical range 1-100 $\mathrm{g} \mathrm{m}^{-3}$ ). Only a tenth of this dose, however, was necessary to eliminate one of the toxin variants which was being studied. It can only be assumed that the two uncharacterised toxins were different microcystin variants which exhibited marked differences in sorption behaviour, probably due to their differing polarities. Subsequent studies confirmed that activated carbon can be effective to varying degrees and for most carbon types high doses of PAC are required. Falconer et al. ${ }^{4}$ reported that many of the PACs in their investigation only removed a small percent of the microcystins when used at a dosing rate of $1 \mathrm{~kg} \mathrm{~m}^{-3}$, with at least $50 \mathrm{~kg} \mathrm{~m}^{-3}$ required for many of the carbon types to be effective.

A number of investigations have assessed the suitability of a range of carbons, both PAC and GAC, produced from differing starting materials e.g. wood, coal, peat and coconut. It has, however, been concluded that the surface characteristics and not the parent carbon source were more important in determining 
sorption performance. ${ }^{5}$ Surface characteristics and therefore sorption properties result from both the nature of the starting material and the mode of activation. This study reported variation in microcystin-LR adsorption levels between 20 to $280 \mu \mathrm{g} \mathrm{mg}^{-1}$ depending on the physical characteristics of PAC. It is generally assumed that the greater the available surface area, the greater the adsorption efficiency of an activated carbon. Surface area is typically determined using the Brunauer, Emmett and Teller (BET) method but this was not found to correspond to the effectiveness of microcystin-LR removal. Previous work on humic acids had suggested that the volume of pores in the mesopore range (2-50 nm diameter) was the main factor influencing their adsorption. Likewise, adsorption of microcystin-LR (estimated diameter 1.2-2.6 nm) was greatest for carbon types which were shown to have high mesopore volumes and this would appear to be the dominant factor influencing adsorption. No correlation was found between micropore volume (diameter $<2 \mathrm{~nm}$ ) and adsorption which suggests toxin molecules are too large to enter these pores hence preventing adsorption.

Competitive adsorption can also greatly influence the amount of microcystin removed by activated carbon. The removal of microcystins in high purity water (e.g. Milli-Q) was compared to that in raw water. In each case, as might be expected, removal efficiencies decline due to competition for binding sites on the carbon. Problems of high natural organic matter (NOM) are particularly relevant to microcystin removal since their presence in water is accompanied by the occurrence of the many components of cyanobacterial cells. Considering that microcystins only account for around, at most, $1 \%$ of the dry weight of cyanobacterial cells, it is only to be expected that the death of a bloom and subsequent release of microcystins will be associated with high carbon loading of the water.

The incorporation of GAC filtration beds is increasingly common in modern treatment works, however, the cost and effort of recharging is high. This reduces the willingness to recharge the beds at relatively short intervals (i.e. months compared to years). It is therefore important to determine GAC capacity and hence breakthrough of toxin into drinking water. It has been demonstrated that breakthrough can occur after filtration of a relatively small water volume. ${ }^{4}$ When a $70 \mathrm{~g} \mathrm{GAC}$ column was utilised, a number of the carbon types tested removed less than $50 \%$ of the toxicity after $10 \mathrm{dm}^{3}$ had been filtered, and even the most efficient material failed after only 50 $\mathrm{dm}^{3}$. This suggests that the useful life of GAC for removal of microcystins is relatively short. Furthermore, tests carried out with GAC which had been used in a water treatment plant for 5 months prior to evaluating adsorption of microcystin, revealed the adsorption capacity $\left(K_{\mathrm{f}}\right)$ of the used carbon was reduced to 6.2 compared with 50 for virgin GAC of the same type. ${ }^{6}$

Some products are available for the treatment of water by the consumer. These include filter jug systems which filter water through a pre-packed cartridge which contains a mixture of GAC along with ion exchange resin. It was found that with some filter types, percentage removal was highly variable and the performance of the filters declined with increasing volume. ${ }^{7}$ None of the filters removed all of the microcystin present in the water. It is not clear which component of the cartridge is removing the microcystin although it could be expected that the GAC adsorbs at least some of the toxins. This is one of the only studies to compare the removal of a number of microcystins and it was consistently found that a slightly greater percentage of the more hydrophobic microcystins (e.g. microcystin-LW) was removed compared with microcystin-LR.

The findings discussed demonstrate that activated carbon can be successfully employed to ensure microcystin removal, however performance may be reduced with normal water treatment practices. Furthermore, little is known about the fate of microcystins adsorbed onto activated carbon. It may be biodegraded and hence rendered safe or it may be desorbed over time with associated hazards to health.

\subsection{Reverse osmosis}

Reverse osmosis (RO) has been used in water treatment for the desalination of brackish or estuarine waters to provide a fresh potable supply. RO is similar to filtration methods in that it separates a liquid from a mixture of suspended or dissolved components. The two methods do, however, differ in their mode of operation. Filtration excludes components from the filtrate due to their size, whereas the primary function of the RO membrane is to exclude dissolved salts through the use of a semi-permeable membrane although it does exclude organic compounds with a molecular weight above 100. As RO is currently in use in a number of countries to provide drinking water, the ability of this process to remove microcystins has also been assessed.

It can be assumed that microcystins will be retained by RO membranes since the molecular weight of microcystins is around 1000. Studies with microcystin-LR and -RR gave retention rates greater than $95 \% .{ }^{8}$ Nodularin, a closely related pentapeptide produced by the brackish water cyanobacteria Nodularia spumigina, was also retained during RO water treatment. ${ }^{9}$ As the salt and toxin concentration increased in the retentate, traces of nodularin were, however, detected in the treated water. This was paralleled by an increase in salinity of the permeate, which was deemed to be a good indicator of water quality. Since brackish waters where nodularin occurs may be desalinated by $\mathrm{RO}$ to provide drinking water, it is reassuring that correctly operated systems will also remove this potential health risk.

Although RO appears to be a suitable method of producing safe drinking water, it must be remembered that this process is also retaining toxin enriched water which has to be disposed safely since the toxins are not destroyed by this treatment.

\section{Chemical treatment methods for the removal of microcystins}

The first report of chemical treatment of microcystins was in 1976 when Hoffmann ${ }^{3}$ studied the effect of a range of reagents on these toxins. Since then there have been several investigations on this topic using a variety of oxidising agents which are commonly employed for treating potable water. Table 1 displays the most frequently used water treatment reagents together with their oxidation potentials in decreasing order of reactivity.

Table 1 Oxidation potentials of chemical oxidants commonly used by the water industry

\begin{tabular}{ll}
\hline Reagent & Oxidation Potential/Volts $v s$. NHE \\
\hline Hydroxyl Radical & 2.80 \\
Ozone & 2.07 \\
Hydrogen Peroxide & 1.78 \\
Perhydroxyl Radical & 1.70 \\
Permanganate & 1.68 \\
Hypochlorous Acid & 1.49 \\
Chlorine & 1.36 \\
Chlorine Dioxide & 1.28 \\
\hline
\end{tabular}

A common feature of the toxin destruction work reported in the literature is a lack of characterisation of decomposition products. The effectiveness of a treatment method tends to be reported in terms of how quickly the parent microcystin disappears. Occasionally toxicity testing by mouse or brine 
shrimp bioassay is performed but frequently the investigation goes no further. A few researchers have carried out mass spectral (MS) analysis of products, but there has been little attempt to rationalise mechanisms for the treatment processes.

Most of the reagents listed in Table 1 will react readily with unsaturated bonds such as those included in the ADDA moiety of microcystin. This group is commonly used as a chromophore for analysis of the toxin (UV absorption $238 \mathrm{~nm}$ ). Therefore if it is modified the parent toxin will seem to disappear. This does not, however, provide information on the by-products that are generated and the potential hazards that may be presented by these materials. The main treatment methods are considered below together with mechanisms that we propose as the probable initial modes of attack on the toxin. These mechanisms are only tentative since there is little detail available in the literature on decomposition products in order to validate our proposals. Much further work, therefore, will have to be performed in order to determine their validity.

\subsection{Chlorination}

Chlorine has been used as a reagent for water treatment since the last century, replacing sand filtration as the disinfection method for potable water in the United States at the beginning of the 20th century. Chlorine dissolves in water forming hypochlorous acid. Above $\mathrm{pH} 5$ this starts to dissociate forming hypochlorite ions, with $100 \%$ dissociation above $\mathrm{pH} 10$. The undissociated hypochlorous acid molecule is established as the most effective disinfecting agent.

Initial work on the application of chlorination for the removal of microcystins carried out by Hoffmann, ${ }^{3}$ Keijola et al. ${ }^{10}$ and Himberg et al. ${ }^{11}$ indicated that the process was ineffective. This may have been due to the work being performed at a $\mathrm{pH}$ where the free chlorine concentrations were relatively low. Although Keijola and Himberg did not report the $\mathrm{pH}$ levels, Hoffman performed his investigation at $\mathrm{pH} 8.5$. The effect of $\mathrm{pH}$ on chlorination of microcystins was subsequently investigated and a dependence on destruction efficiency was observed. ${ }^{12}$

Nicholson et al. investigated the use of chlorine and chloramine for the destruction of microcystin and the pentapeptide toxin nodularin. ${ }^{12}$ Both toxins were destroyed provided a residual level of $0.5 \mathrm{mg} \mathrm{dm}^{-3}$ chlorine was maintained for 30 minutes. A $\mathrm{pH}$ dependence was observed with $\mathrm{NaOCl}$ and $\mathrm{Ca}(\mathrm{OCl})_{2}$ being less effective at higher $\mathrm{pH}$ values. Table 2

Table 2 Destruction efficiencies of chlorinating agents for the destruction of microcystin-LR

\begin{tabular}{lll}
\hline Reagent & Concentration $/ \mathrm{mg} \mathrm{dm}^{-3}$ & $\begin{array}{l}\% \text { Destruction after } \\
30 \text { minutes treatment }\end{array}$ \\
\hline Aqueous $\mathrm{Cl}_{2}$ & 1 & $>95$ \\
$\mathrm{Ca}(\mathrm{OCl})_{2}$ & 1 & $>95$ \\
$\mathrm{NaOCl}$ & 1 & $\sim 40$ \\
$\mathrm{NaOCl}$ & 5 & $70-80$ \\
\hline
\end{tabular}

shows the relative efficiencies of chlorinating agents for the destruction of toxin concentrations in the range of 130-300 $\mu \mathrm{g} \mathrm{dm}^{-3}$ which represents highly contaminated water.

The rates of toxin destruction were greatly reduced at $\mathrm{pH}$ levels above 8 varying from $79 \%$ at $\mathrm{pH} 7$ to $0.4 \%$ at $\mathrm{pH} 10$. This was believed to be due to decreasing concentrations of hypochlorous acid which is a more effective oxidant than the hypochlorite ion. No by-products of destruction were reported or mechanism for the process discussed. Chloramination was found to be an ineffective treatment for both toxins with little effect on the level of either compound after a 5 day treatment with a $20 \mathrm{mg} \mathrm{dm}-3$ chloramine solution.
Tsuji et al. examined the effect of sodium hypochlorite on microcystin-LR and -RR. ${ }^{13}$ They found that the toxin disappeared with little difficulty and was dependent on the free chlorine dose. Following a 30 minute treatment $99 \%$ toxin removal was observed for free chlorine concentration of 2.8 $\mathrm{mg} \mathrm{dm}-3$, while only $35 \%$ destruction was achieved at a level of $0.7 \mathrm{mg} \mathrm{dm}^{-3}$ with contact time of 60 minutes. Several reaction products were observed by HPLC one of which was identified as a dihydroxy-microcystin. They proposed that this compound was generated by the action of the free chlorine on either of the unsaturated bonds of the ADDA group, to form a dichloromicrocystin, followed by hydrolysis. Stereo- and regioisomers may also have been produced. The formation of the dichloromicrocystin postulated by this group is an unlikely intermediate of decomposition. It is thought that the more plausible mechanism to occur is that the halogen dissolves in water forming hypochlorous acid. Hypochlorous acid may in turn be protonated and become a powerful electrophile which subsequently reacts with unsaturated bonds to form a chlorohydrin. The halogen in the chlorohydrin may then undergo a nucleophilic substitution reaction with the solvent to form a dihydroxy-microcystin (Scheme 1).

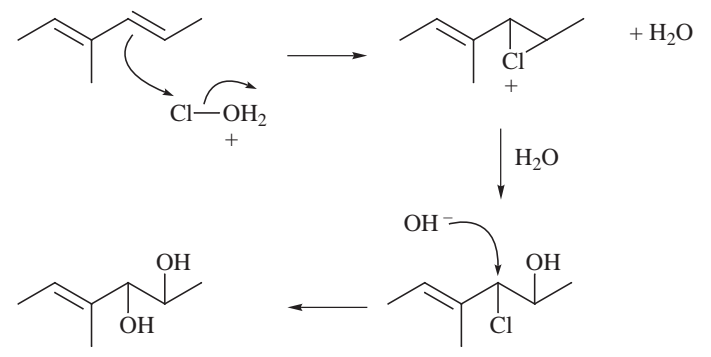

Scheme 1 Decomposition of microcystin-LR via chlorination.

To date there have been no reports of the use of chlorine dioxide for the treatment of microcystins. This has been applied effectively to the treatment of many organic compounds and it is believed that it may have applications in the destruction of microcystins.

Chlorination does appear to provide an effective method of removing microcystins from potable water although this is dependant on dose and the maintenance of adequate residual chlorine levels. Unfortunately the mechanism and products of decomposition remain to be fully characterised and therefore the possible formation of harmful by-products is as yet unknown.

\subsection{Ozonation}

Ozone is an unstable gas with a relatively high oxidation potential and is used for water treatment by dispersing the gas in aqueous media. It is widely utilised for treating drinking water but it is an expensive and sometimes unpredictable reagent. Ozone combined with UV light has proved to be an effective reagent and is very efficient in destroying a wide range of organic compounds.

Ozone is highly reactive towards double bonds, forming carbonyl compounds via ozonides. Either of the double bonds in the ADDA group in microcystin would be susceptible to such an attack so decomposing the parent toxin (Scheme 2). The toxicity of microcystins has been shown to be associated with the ADDA olefin groups, therefore cleavage of this moiety would be expected to eliminate toxicity. Harada et al. ${ }^{14}$ used ozone to cleave one of the double bonds on the ADDA group which would support this hypothesis.

Early studies by Keijola et al. ${ }^{10}$ reported a $100 \%$ efficiency of $1 \mathrm{mg} \mathrm{dm}^{-3}$ ozone for the removal of up to $60 \mu \mathrm{g} \mathrm{dm}^{-3}$ microcystin. 


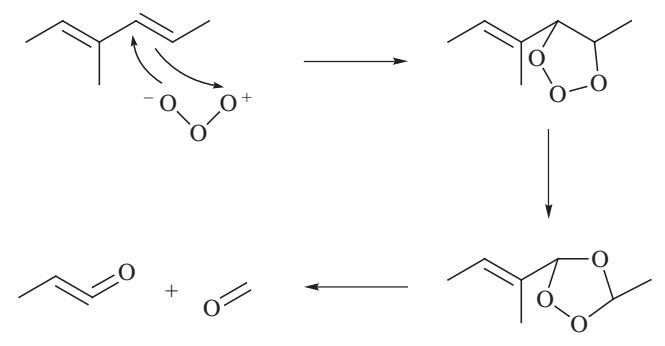

Scheme 2 Decomposition of microcystin-LR by ozone.

Rositano and co-workers have found that $99 \%$ of microcystin was removed in 15 seconds when treated with $0.05 \mathrm{mg} \mathrm{dm}^{-3}$ ozone. ${ }^{15}$ Their investigations found that ozone was more effective than chlorine, hydrogen peroxide and potassium permanganate for the destruction of microcystin-LR, which corresponds to the higher oxidation potential of ozone. They found combining with hydrogen peroxide further enhanced the efficiency of ozone treatment. A pH dependence on the destruction efficiency was observed with alkaline conditions being less favourable. This was rationalised by the fact that the oxidising potential of ozone is 1.24 volts vs. NHE (normal hydrogen electrode) in basic solutions compared to 2.07 volts $v s$. NHE in acidic solutions.

The reported studies all suggest that ozonation is an effective method for the removal of microcystins from drinking water. Although it is still important to characterise the decomposition products and their potential health implications, the overall effectiveness of ozonation as a suitable water purification method appears promising.

\subsection{Permanganate}

The large scale application of permanganate began in the 1960s in the United States and Europe. It is a strong oxidising agent capable of destroying organic compounds and micro-organisms.

Permanganate generally attacks functional groups with multiple bonds and will cleave benzene rings. Permanganate is commonly used by organic chemists for the hydroxylation of alkenes with the formation of diols (Scheme 3 ). It should

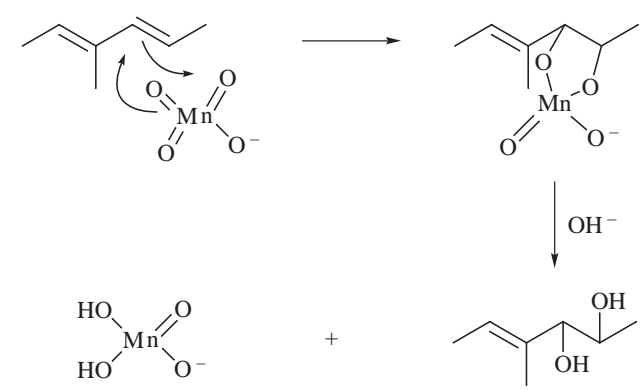

Scheme 3 Decomposition of microcystin-LR by permanganate.

therefore be effective in decomposing microcystin and removing the toxicity by attacking the unsaturated bonds in the ADDA group.

A $1 \mathrm{mg} \mathrm{dm}-3$ solution of potassium permanganate removed 95\% of a $200 \mu \mathrm{g} \mathrm{dm}-3$ microcystin-LR solution in 30 minutes. ${ }^{15}$ A comparison of the oxidation of microcystin-LR using chlorine and potassium permanganate (both at 2 $\mathrm{mg} \mathrm{dm}{ }^{-3}$ ) demonstrated that the toxin followed a similar decay curve for both oxidants. Permanganate however, was observed to bring about more rapid removal of the microcystin which is consistent with the known oxidation potentials (Table 1). No by-products of decomposition were characterised.

This reagent appears to show much promise but there has been little detailed study of the system for microcystin destruction.

\subsection{Hydrogen peroxide}

The use of hydrogen peroxide in water treatment is limited. Although thermodynamic data suggest that this should be an effective oxidant (Table 1), the kinetics for many water treatment applications are unfavourable. Hydrogen peroxide has, however, been applied to the oxidation of phenolic wastewater and for the treatment of paper mill effluent, drilling muds and other types of organic wastewater.

The oxidising effectiveness of hydrogen peroxide can be enhanced by irradiating it with UV light. The light dissociates the molecule generating two highly reactive hydroxyl radicals. A range of organics including aromatics, alcohols, aliphatics and haloaliphatics has been successfully treated using $\mathrm{UV} / \mathrm{H}_{2} \mathrm{O}_{2}$.

Research has shown that hydrogen peroxide is relatively ineffective in degrading microcystin-LR with only $17 \%$ removal after 60 minutes treatment with a $20 \mathrm{mg} \mathrm{dm}^{-3}$ solution of peroxide. ${ }^{16}$ Further work investigated the effect of a $2 \mathrm{mg} \mathrm{dm}^{-3}$ hydrogen peroxide solution on $1 \mathrm{mg} \mathrm{dm}^{-3}$ solution of microcystin-LR. ${ }^{15}$ Virtually no toxin destruction was observed after 10 minutes. Combination of $\mathrm{H}_{2} \mathrm{O}_{2}$ with ozone was, however, extremely effective with virtually all the toxin being removed within 30 seconds. There are no reports of the use of $\mathrm{UV} / \mathrm{H}_{2} \mathrm{O}_{2}$ for the treatment of microcystin-containing water.

This reagent shows little promise as an effective treatment method for microcystins although there may be scope for the use of combined treatments such as ozone $/ \mathrm{H}_{2} \mathrm{O}_{2}$ which has already been shown to be very powerful and $\mathrm{UV} / \mathrm{H}_{2} \mathrm{O}_{2}$ which requires research to determine its suitability for microcystin removal.

\subsection{Photolysis}

Photolytic oxidation can occur by a number of methods. To bring about photodegradation it is necessary that the compound absorbs light at a wavelength which is the same as that emitted by the light source. If this is not the case the photolytic breakdown may still be achieved using a sensitiser or relay which will absorb the light and initiates the decomposition reaction.

In many cases there is an electron transfer from the excited state of the molecule undergoing degradation to ground state oxygen, generating the superoxide radical anion. ${ }^{17}$ The radical cation formed by this process may then be hydrolysed. In some cases, for example the photolytic degradation of halogenated aliphatic and aromatic compounds, radicals may form via homolysis which subsequently react with oxygen. This process has been applied to a wide range of organic substrates including chlorinated aromatics and aliphatics, nitrotoluenes, phenols, and oil products.

A number of research groups have examined the photolysis of microcystins although few have attempted to provide any mechanistic explanation for the process. ${ }^{18-21}$.

The main chromophores in the molecule are in the ADDA group and are utilised in the analytical methods. These groups are the aromatic ring and the unsaturated bonds at the 4-5 and 6-7 positions. When an electron is promoted to a $\pi^{*}$ orbital in the unsaturated bond the $\pi$ bond will uncouple leaving only the $\sigma$ bond between the carbon atoms. The electrons in the carbon $2 p$ orbitals will repel each other and to minimise this a rotation 
about the single bond may occur. This mechanism is believed to be the process involved in the cis-trans photoisomerisation in olefins. Photoisomerisations of microcystins have been reported by Tsuji et al. and Kaya and Sano. ${ }^{18,19}$

Cycloaddition reactions with the unsaturated bond are possible where the weakened $\pi$ bond in the excited state forms two $\sigma$ bonds with a suitable substrate. An internal cycloaddition on the ADDA grouping would be possible between the unsaturated bonds and the aromatic ring. It is important to consider the geometry of the ADDA group on the microcystin molecule. It has been proposed that this group is U-shaped and curled over the microcystin structure. ${ }^{22}$ Such a geometry would bring the benzene ring and double bonds into close proximity with one another (Scheme 4). This type of internal cycloaddi-

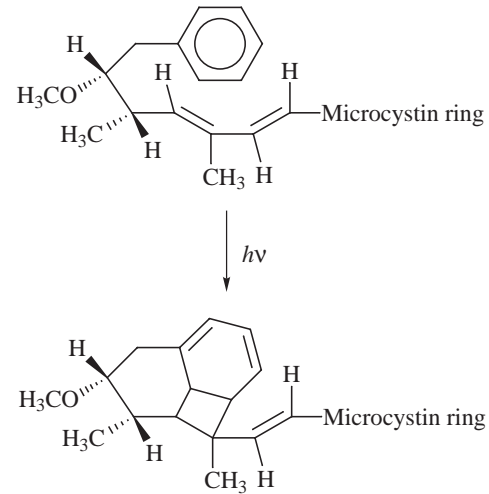

Scheme 4 Internal photosensitised cycloaddition in microcystin-LR.

tion has recently been reported for microcystin-LR. ${ }^{19}$ It should be noted however that a number of other reports have suggested a solution structure where the ADDA group is not bent in such a fashion. ${ }^{23,24}$ This suggests that the geometry is not appropriate for an internal cycloaddition. However, Bagu found that the ADDA side chain is sufficiently flexible ${ }^{23}$ that in principle it could momentarily position itself favourably for the cycloaddition to occur.

On photoexcitation microcystin could transfer an electron to molecular oxygen generating a microcystin radical cation and a superoxide radical anion. These species may then react with one another so generating oxidation products such as carbonyl compounds. The radical cation of the toxin could also form oxidation products by reaction with molecular oxygen. It is interesting to note that superoxide reacts 30 times faster with olefin radical cations than does molecular oxygen. ${ }^{25}$ In the aqueous systems superoxide is rapidly protonated ${ }^{26}$ generating the hydroperoxide radical. This radical may attack the double bonds of microcystin, generating hydroperoxide products.

In the presence of suitable sensitisers singlet oxygen may also be generated. Singlet oxygen is highly reactive towards unsaturated bonds generating peroxides, hydroperoxides, dioxetanes and carbonyl compounds. ${ }^{27}$

Scheme 5 displays speculative mechanisms for the photochemical oxidation of microcystin in aqueous solution. Tsuji et al. investigated the effect of sunlight on microcystin-LR and -RR. ${ }^{18}$ Irradiation alone had little effect on either microcystin with $86 \%$ of the toxin remaining after 26 days photolysis. Addition of naturally photosynthetic pigments greatly enhanced the destruction process with over $95 \%$ decomposed following 29 days photolysis. The rate of destruction depended on the pigment concentration. A photo-induced geometric isomerisation of microcystin to a less toxic isomer was also reported. At higher pigment concentrations the rate of decomposition was found to be faster than the rate of isomerisation. The pigments involved in the photolytic process are phycocyanins which on photolysis in oxygenated solutions generate both singlet oxygen

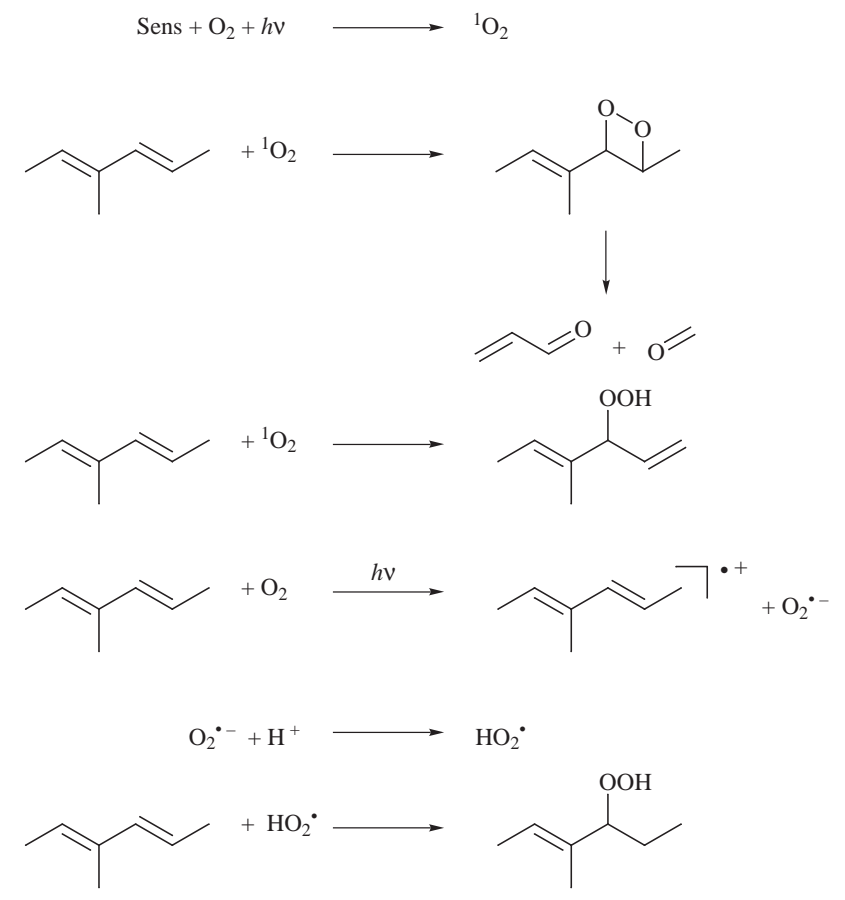

Scheme 5 Potential modes of photochemical decomposition of microcystinLR.

and superoxide. ${ }^{28}$ It has been proposed that these molecules catalyse the toxin destruction via the generation of both singlet oxygen and superoxide which subsequently forms peroxide and hydroxyl radicals. ${ }^{21}$ Tsuji reported from MS data that a dihydroxy-microcystin had formed resulting from hydroxyl radical attack on the diene group of the ADDA. This would be consistent with an electron transfer type mechanism with the eventual formation of peroxide which subsequently disproportionates forming hydroxyl radicals.

Further work by Tsuji et al. showed that microcystin-LR could be completely destroyed within 10 minutes under a UV light intensity of $2550 \mathrm{~W} \mathrm{~cm}^{-2} .{ }^{28}$ When a weaker light source was used isomerisation was again observed.

Welker and Steinberg have studied the effect of humic substances in the indirect photolysis of microcystins-LR, -YR and -RR. ${ }^{29}$ Following 8 hours photolysis the concentrations of these toxins were 5,53 and $44 \%$ of the initial concentration. The active agents for the decomposition process were again believed to be singlet oxygen, hydrogen peroxide and hydroxyl radical generated by the humic substances.

Kaya and Sano reported the formation of three major nontoxic products when microcystin-LR was irradiated with UV light. ${ }^{19}$ Two of these products were geometric isomers of the ADDA group of the toxin, [4(E),6(Z)-ADDA] and $[4(Z), 6(E)-$ ADDA] microcystin. The third product was a previously unidentified tricyclo-ADDA-microcystin formed by a [2+2] addition between the aromatic ring and the unsaturated bond at the 6-7 position of the ADDA group of the microcystin. While the isomeric products were stable to further photolysis the cyclic product degraded although the products of this decomposition were not identified. The authors suggested that the photolytic destruction of microcystin proceeded via the tricyclo-ADDA-microcystin-LR. UV irradiation resulted in a $50 \%$ reduction in concentration of microcystin-LR in 75 minutes.

Much of the work on photolysis is inapplicable for the removal of microcystin from potable supplies. The production of modified microcystins in the natural environment through the actions of photosensitising compounds like humic acids and pigments gives valuable insight into breakdown/detoxification mechanisms. It also identifies other microcystin related compounds which may be entering water treatment systems from natural raw waters. 


\subsection{Semiconductor photocatalysis}

The use of semiconductor photocatalysis for the destruction of environmental pollutants is a well established technique with the mineralisation of a wide range of materials being reported. Semiconductors have a band structure with a filled valence band and an empty conductance band separated by a band gap. When semiconductors (SC) are illuminated with light of energy greater than the band gap, electrons are promoted from the occupied valence band to the unoccupied conductance band (Fig. 2). This generates oxidising sites in the valence band and

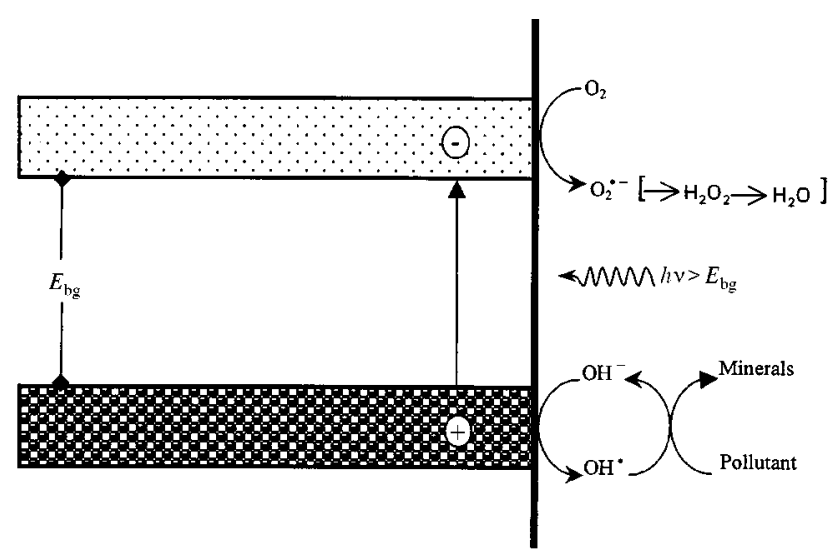

Fig. 2 The process of semiconductor photocatalysis for water purification.

reducing sites in the conductance band. It is believed that preadsorbed hydroxide ions are oxidised to hydroxyl radicals at the valence band. These radicals subsequently oxidise the polluting material, while at the conductance band an electron is donated to oxygen thereby generating the superoxide radical anion. The superoxide is then protonated and eventually forms hydrogen peroxide which also acts as an oxidising agent.

The use of a titanium dioxide photocatalyst $(1 \% \mathrm{w} / \mathrm{v})$ for the removal of microcystin-LR in water has been investigated. ${ }^{30} \mathrm{It}$ was found that even at levels of $200 \mathrm{mg} \mathrm{dm}^{-3}$ the toxin rapidly degraded when illuminated in the presence of the catalyst. The process appeared to follow Langmuir-Hinshelwood kinetics although a discrepancy was observed between the adsorption constants determined for the photocatalytic process and those obtained from dark isotherms. No breakdown was observed in light alone.

A subsequent study investigated the factors which influenced the rate of the toxin destruction at the photocatalyst surface. ${ }^{31} \mathrm{~A}$ primary kinetic isotope effect of approximately 3 was observed when the destruction was performed in a heavy water solvent. Hydroxylated compounds were observed as products of the destruction process. No destruction was observed when the process was investigated under a nitrogen atmosphere. Oxygen must be present in order to react with electrons in the conductance band and products of this reduction, e.g. peroxide, may be involved in the destruction of microcystin.

A more detailed mechanistic study of the photocatalytic destruction of microcystin showed that the toxin disappearance was accompanied by the appearance of seven UV detectable compounds. ${ }^{32}$ Spectral analysis revealed that some of these compounds retained spectra similar to the parent compound suggesting that the ADDA moiety, thought to be responsible for the characteristic spectrum, remained intact, whereas the spectra of some of the other products were more radically altered. Six of the seven observed reaction products did not appear to undergo further degradation during prolonged photocatalysis (100 min). The degree to which microcystin-LR was mineralised by photocatalytic oxidation was determined, and it was found that less than $10 \%$ mineralisation occurred.
Since more than $90 \%$ of the toxin is not mineralised it is important to fully characterise the products of decomposition and assess their health impact.

Shepard et al. ${ }^{33}$ have also reported the use of $\mathrm{TiO}_{2}$ photocatalysis for the degradation of microcystin-LR and two other microcystins, YR and YA. Rapid decomposition was observed with a half life of less than 5 minutes for each toxin.

These findings suggest that photocatalytic destruction of microcystins may be a suitable method for the removal of these potentially hazardous compounds from drinking water. As for all the other treatment processes detailed characterisation of the decomposition by-products is necessary.

\section{Conclusion}

The water treatment methods reviewed here could be applied to the removal of microcystins with varying degrees of ease and effectiveness. A number of oxidative methods have been discussed and although most appear to be suitable many exhibit limitations. Chlorination, a well established technique, may appear effective but the relatively high dosing rates and contact time required is outside commonly adopted protocols. Its use would therefore have to be carefully monitored and it is essential that further work is carried out to determine the potential hazards associated with by-products. Ozonation is very effective for the rapid removal of cyanotoxins from potable water and could provide an appropriate treatment method. Cost may prove to be prohibitive particularly since contamination with microcystins is typically seasonal and unpredictable. Of the other oxidative methods discussed, photocatalytic degradation does appear to be very promising. This is a relatively new treatment method and it remains to be seen how well it will perform but initial findings are very favourable. Its great strengths are that application should be relatively simple and easy to operate, it should mineralise most organics with a limited chance for the production of harmful by-products, and it is potentially a sustainable and clean technology.

Both established and new advanced technologies appear to render microcystin contaminated water safe to drink. It is important however that a greater effort is made to understand the mechanisms by which the disappearance of microcystins is achieved. Furthermore, work must be done to structurally characterise the breakdown products generated from chemical treatment methods. Where possible the effects of acute and chronic exposure to common by-products should be carried out. By their nature, methods which physically remove microcystins can be used without concern about by-product generation, but their safe use must be monitored closely to ensure systems are functioning as expected. It was lack of vigilance in the use of both GAC and RO that allowed over 50 dialysis patients in Brazil to die as a result of microcystin exposure ${ }^{2}$.

Most work has centred on microcystin-LR. Considering there are over 60 different variants of microcystin it will be important to investigate the suitability of each process on representative variants.

The effective use of physico-chemical methods will be hampered by increased NOM associated with cyanobacterial blooms. This will greatly reduce the efficiency of the treatment processes due to competition for active species or binding sites.

\section{References}

1 Toxic Cyanobacteria in Water, Eds. I. Chorus and J. Bartram, E \& FN Spon, London, 1999 
2 S. Pouria, A. deAndrade, J. Barbosa, R. L. Cavalcanti, V. T. S. Barreto, C. J. Ward, W. Presser, G. K. Poon, G. H. Neild and G. A. Codd, The Lancet, 1998, 352, 21.

3 J. R. H. Hoffman, Water SA, 1976, $2,58$.

4 C. Donati, M. Drikas, R. Hayes and G. Newcombe, Water Res., 1994, 28, 1735 .

5 I. R. Falconer, M. T. C. Runnegar and V. L. Huynh, Tenth Federal Convention of the Australian Water and Wastewater Association, Sydney, 1983, p. 26-1.

6 T. W. Lambert, C. F. B. Holmes and S. E. Hrudey, Water Res., 1996, 30, 1411.

7 L. A. Lawton, B. J. P. A. Cornish and A. W. R. MacDonald, Water Res., 1998, 32, 633.

8 U. Neumann and J. Weckesser, Environ. Toxicol. Water Qual., 1998, 13, 143.

9 E. Vuori, A. Pelander, K. Himberg, M. Waris and K. Niinivaara, Water Res., 1997, 31, 2922.

10 A. M. Keijola, K. Himberg, A. L. Esala, K. Sivonen and L. Hiisvirta, Toxic. Assess., 1988, 3, 643.

11 K. Himberg, A. M. Keijola, L. Hiisvirta, H. Pyysalo and K. Sivonen, Water Res, 1989, 23, 979.

12 B. C. Nicholson, J. Rositano and M. D. Burch, Water Res, 1994, 28, 1297.

13 K. Tsuji, T. Watanuki, F. Kondo, M. Watatabe, S. Suzuki, H. Nakazawa, M. Suzuki, H. Uchida and K. Harada, Toxicon, 1997, 35, 1033.

14 K.-i. Harada, H. Murata, Z. Quiang, M. Suzuki and F. Konda, Toxicon, 1996, 34, 701.

15 J. Rositano, B. C. Nicholson, P. Pieronne, Ozone Sci. Technol., 1998, 20, 223.

16 M. Drikas, Control and/or removal of toxins, in Toxic Cyanobacteria: Current Status of Research and Management, Eds: D. A. Steffensen and B. C. Nicholson, Australian Centre for Water Quality, Salisbury, Australia, 1994, p. 93.
17 O. Legrini, E. Oliveros and A. M. Braun, Chem. Rev, 1993, 93, 671.

18 K. Tsuji, S. Nalto, F. Kondo, N. Ishikawa, M. F. Watanabe, M. Suzuki and K.-I. Harada, Environ. Sci. Technol., 1994, 28, 173.

19 K. Kaya and T. Sano, Chem. Res. Toxicol., 1998, 11, 159.

20 T. Lanaras, C. M. Cook, J. E. Eriksson, J. A. O. Meriluoto and M. Hotokka, Toxicon, 1991, 901, 906.

21 J. R. Bagu, F. D. Sönnichsen, D. Williams, R. J. Andersen, B. D. Sykes and C. F. B. Holmes, Struct. Biol., 1995, 2, 114.

22 J. Goldberg, H.-B. Huang, Y.-G. Kwon, P. Greengard, A. C. Nairn and J. Kuriyan, Nature, 1995, 376, 745.

23 M. Julliard and M. Chanon, Chem. Rev., 1983, 83, 425.

24 E. R. Carraway, A. J. Hoffmann and M. R. Hoffmann, Environ. Sci. Technol., 1994, 28, 494.

25 C. S. Foote, in Singlet Oxygen: Reactions with Organic Compounds and Polymers, B. Rånby and J. F. Rabek, Eds., John Wiley and Sons, New York, 1978, pp. 135.

26 J.-A. He, Y.-Z. Hu and L.-J. Jiang, Biochim. Biophys. Acta, 1997, 1320, 165.

27 P. K. J. Robertson, L. A. Lawton and B. J. P. A. Cornish, J. Porphyrins Phthalocyanines, 1999, accepted for publication.

28 K. Tsuji, T. Watanuki, F. Kondo, M. Watatabe, S. Suzuki, H. Nakazawa, M. Suzuki, H. Uchida and K. Harada, Toxicon, 1995, 33, 1619.

29 M. Welker and C. Steinberg, Water Res., 1999, 33, 1159.

30 P. K. J. Robertson, L. A. Lawton, B. Münch and J. Rouzade, Chem. Commun., 1997, 4, 393.

31 P. K. J. Robertson, L. A. Lawton, B. J. P. A. Cornish and M. Jaspars. J. Photochem. Photobiol. A. Chem., 1998, 116, 215.

32 L. A. Lawton, P. K. J. Robertson, B. J. P. A. Cornish and M. Jaspars, Environ. Sci. Technol., 1999, 33, 771.

33 G. S. Shepard, S. Stockenstrom, D. De Villiers, W. J. Engelbrecht, E.W. Sydenham and G. F. S. Wessels, Toxicon, 1998, 36, 1895.

Review 8/05416I 